\title{
Enhancing precision in fs-laser material processing by simultaneous spatial and temporal focusing
}

\author{
Robert Kammel $^{1}$, Roland Ackermann ${ }^{1}$, Jens Thomas ${ }^{1,2}$, Jörg Götte ${ }^{3}$, Stefan Skupin ${ }^{3,4,5}$, Andreas Tünnermann ${ }^{1,6}$ \\ and Stefan Nolte ${ }^{1,6}$
}

In recent years, femtosecond (fs)-lasers have evolved into a versatile tool for high precision micromachining of transparent materials because nonlinear absorption in the focus can result in refractive index modifications or material disruptions. However, when high pulse energies or low numerical apertures are required, nonlinear side effects such as self-focusing, filamentation or white light generation can decrease the modification quality. In this paper, we apply simultaneous spatial and temporal focusing (SSTF) to overcome these limitations. The main advantage of SSTF is that the ultrashort pulse is only formed at the focal plane, thereby confining the intensity distribution strongly to the focal volume and suppressing detrimental nonlinear side effects. Thus, we investigate the optical breakdown within a water cell by pump-probe shadowgraphy, comparing conventional focusing and SSTF under equivalent focusing conditions. The plasma formation is well confined for low pulse energies $<2 \mu \mathrm{J}$, but higher pulse energies lead to the filamentation and break-up of the disruptions for conventional focusing, thereby decreasing the modification quality. In contrast, plasma induced by SSTF stays well confined to the focal plane, even for high pulse energies up to $8 \mu \mathrm{J}$, preventing extended filaments, side branches or break-up of the disruptions. Furthermore, while conventional focusing leads to broadband supercontinuum generation, only marginal spectral broadening is observed using SSTF. These experimental findings are in excellent agreement with numerical simulations of the nonlinear pulse propagation and interaction processes. Therefore, SSTF appears to be a powerful tool to control the processing of transparent materials, e.g., for precise ophthalmic fs-surgery.

Light: Science \& Applications (2014) 3, e169; doi:10.1038/Isa.2014.50; published online 9 May 2014

Keywords: filamentation; fs-laser surgery; laser-induced optical breakdown; materials processing; plasma shadowgraphy

\section{INTRODUCTION}

In recent years, defined energy deposition by focused ultrashort laser pulses has been of increasing interest both in science and for industrial applications, e.g., for precise micro machining. ${ }^{1}$ For example, pulses of several picoseconds (ps) allow material ablation with low thermal side effects for accurate drilling or cutting. ${ }^{2}$ However, for some applications, even shorter pulses of several tens of femtoseconds ( $f$ s) are attractive because high intensities $>10^{13} \mathrm{~W} \mathrm{~cm}^{-2}$ can be reached by focusing such fs-pulses with a comparatively low energy of only several $\mu \mathrm{J}$. When the focus is located within transparent materials such as glasses or tissue, nonlinear absorption processes lead to the formation of a micrometer-sized plasma in the focal volume, initiating a laserinduced optical breakdown. ${ }^{3}$ Depending on the incident intensity, material modifications such as permanent refractive index changes or disruptions can be realized. ${ }^{4,5}$

Especially for ophthalmic purposes, the well-localized nonlinear lasertissue interaction has enabled new surgical techniques such as the wellestablished fs-laser-assisted in situ keratomileusis, where corneal tissue is cut and ablated for refractive correction. ${ }^{6}$ In contrast to solid-state materials, however, biological tissue consists primarily of water, and the laserwater interaction significantly contributes to the induced modifications. ${ }^{7}$ Thus, for sufficient pulse energy, the laser-induced plasma transfers its energy to the surrounding material within several ps, where the temperature rise causes the vaporization of the tissue. Finally, a cavitation bubble is formed, which leads to the local disruption of the transparent tissue. $^{7}$

New therapeutic approaches aim at the treatment of the central and posterior segment of the eye. ${ }^{8}$ For example, in fs-laser cataract surgery, ultrashort laser pulses of several $\mu \mathrm{J}$ are currently used for the rhexis of the lens capsule and for the fragmentation of the cataractous lens tissue to simplify its extraction. ${ }^{9,10}$ A similar strategy is applied in the concept of fs-laser presbyopia treatment, which aims to restore the elasticity of the lens by inducing laser disruptions in the hardened lens nucleus. ${ }^{11-13}$ Moreover, fs-laser-induced cuts within the vitreous body may reduce vitreous traction and are therefore discussed as a preventive therapy for posterior vitreous detachment. ${ }^{14}$

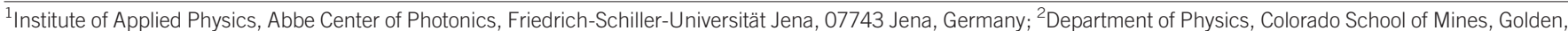

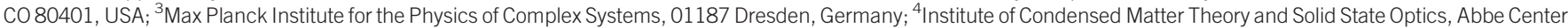

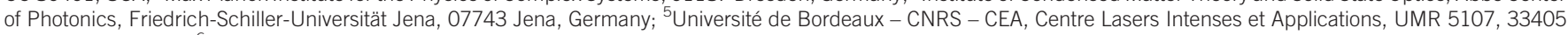
Talence, France and ${ }^{6}$ Fraunhofer Institute for Applied Optics and Precision Engineering, 07745 Jena, Germany

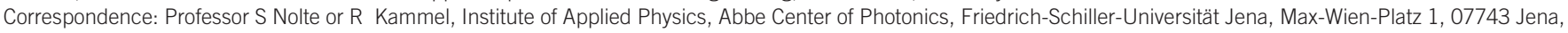
Germany

E-mail: stefan.nolte@uni-jena.de; robert.kammel@uni-jena.de

Received 27 August 2013; revised 24 January 2014; accepted 15 February 2014 
When treating the central or posterior segment of the eye, its anatomy requires focusing with a relatively low numerical aperture (NA) of $\sim 0.1$. Thus, the long propagation distance of the pulse within the ocular medium and the high peak intensities initiate nonlinear side effects. ${ }^{15,16}$ When the pulse energy exceeds a critical power $P_{\text {crit }}$, a dynamical balance between Kerr self-focusing and the defocusing effect of the induced plasma may result in the formation of an elongated filament along the propagation axis. ${ }^{17}$ Moreover, nonlinear frequency conversion, due to self-phase modulation, self-steepening of the pulse and sharp plasma gradients, produces a broadband supercontinuum (SC) ${ }^{18}$ All such nonlinear side effects are detrimental because the increased breakdown length reduces the quality of surgical cuts or even harms tissue outside the focal spot, while extended cut patterns of enlarged, persistent disruptions decrease the visual quality of the eye. ${ }^{19}$ Additionally, retinal safety limits may be affected due to the strong broadband SC generation.

So far, several studies have investigated laser-induced optical breakdown and its side effects such as self-focusing and filamentation in condensed matter. ${ }^{20-22}$ Investigations of the breakdown formation in biological tissue have revealed a strong increase in nonlinear side effects for NA $<0.5 .^{23}$ Thus, the formation of $>100 \mu \mathrm{m}$ streaks inside the tissue of laser-treated rabbit corneas was detected for rather low pulse energies of $1 \mu \mathrm{J},{ }^{15}$ whereas higher pulse energies even hampered a precise energy deposition due to strong self-focusing in a tissue model. $^{14}$

To overcome these undesired side effects, varying the pulse duration along the direction of propagation by simultaneous spatial and temporal focusing (SSTF) is a promising approach. ${ }^{24,25}$ In this concept, the spectral components of the incident ultrashort laser pulse are spatially separated, resulting in a rainbow-like collimated beam, while reducing the local bandwidth within this beam leads to an increased pulse duration. Downstream from the focusing optics, the previously separated spectral components start to overlap. Within the focal volume, the entire bandwidth is recovered, leading to the reconstruction of the initial ultrashort pulse duration. In addition to the minimization of nonlinear effects, the intensity in front of the focus is reduced owing not only to the geometrical change of the beam diameter but also to the change of the pulse duration. Consequently, the nonlinear laser-material interaction is more strongly confined to the focal volume than for conventional focusing.

Until now, the concept of SSTF has been used for various applications, mainly focusing pulses with low energy and high NA at high repetition rates, e.g., in nonlinear fluorescence microscopy. ${ }^{25-27}$ In addition to the advantage of an improved axial resolution, the strong intensity confinement of SSTF enabled the precise ablation of transparent materials and the generation of circular microfluidic channels in glass. ${ }^{28,29}$ Furthermore, the suitability of SSTF for tissue ablation by laser-induced optical breakdown has recently been shown. ${ }^{30,31}$

In this paper, we present detailed experimental and numerical investigations of the nonlinear pulse propagation and breakdown formation induced by SSTF and conventional focusing at low repetition rate. Special emphasis is put on possible applications of SSTF in ophthalmic fs-laser surgery. Here, water is a commonly accepted model system for experiments on laser-tissue interaction due to its high content in biological tissue. ${ }^{7}$ We therefore analyze plasma formation and subsequent disruptions as well as nonlinear side effects such as filamentation in a water cell through pump-probe shadowgraphy. While previous studies of SSTF were frequently based on strongly elliptical beams caused by spectral separation, ${ }^{30,32-34}$ this study emphasizes equivalent focusing parameters such as a comparable
NA to compare the nonlinear pulse-material interaction induced by conventional focusing and SSTF. This factor is of special importance because several applications, including ophthalmic laser surgery, require certain focusing conditions, where, for example, the NA is limited by the pupil size of the eye. Beside the analysis of optical breakdown, a measurement of the spectral broadening by SC generation is presented. Finally, we conclude with a detailed discussion of how SSTF drastically improves the conditions for fs-surgery of the lens or the posterior eye segment.

\section{MATERIALS AND METHODS}

\section{Experimental realization}

To induce and characterize the nonlinear laser-material interaction through conventional focusing and SSTF, a pump-probe set-up is used (Figure 1).

Pulses are delivered by a laser system consisting of a mode-locked oscillator and a chirped pulse amplification laser (Tsunami \& Spitfire; Newport Corporation, Irvine, CA, USA). The system emits pulses with a minimal pulse duration of $\tau_{0}=50 \mathrm{fs}$ and a bandwidth of $\Delta \lambda \approx 30 \mathrm{~nm}$, centered at a wavelength of $\lambda_{0}=800 \mathrm{~nm}$. The output beam diameters are $2 w_{x}=5.2 \mathrm{~mm}$ and $2 w_{y}=5.1 \mathrm{~mm}\left(1 / e^{2}\right)$. The pulse repetition rate of the laser system is set to $200 \mathrm{~Hz}$, which is further reduced to $10 \mathrm{~Hz}$ using a synchronized mechanical chopper (MC2000; Thorlabs Inc., Newton, NJ, USA) to rule out interactions between subsequent breakdown events.

Pulses entering the set-up are split into a pump and a probe beam path. Within the pump-beam path, the pulse energy is adjusted using a half-wave plate and a polarization beam splitter cube. In the SSTF configuration, a cylindrical telescope reduces the horizontal beam width by a factor of 5 to an elliptical beam with a width of $2 w_{x 0}=1.0 \mathrm{~mm}$. Afterward, the elliptical beam is spectrally separated

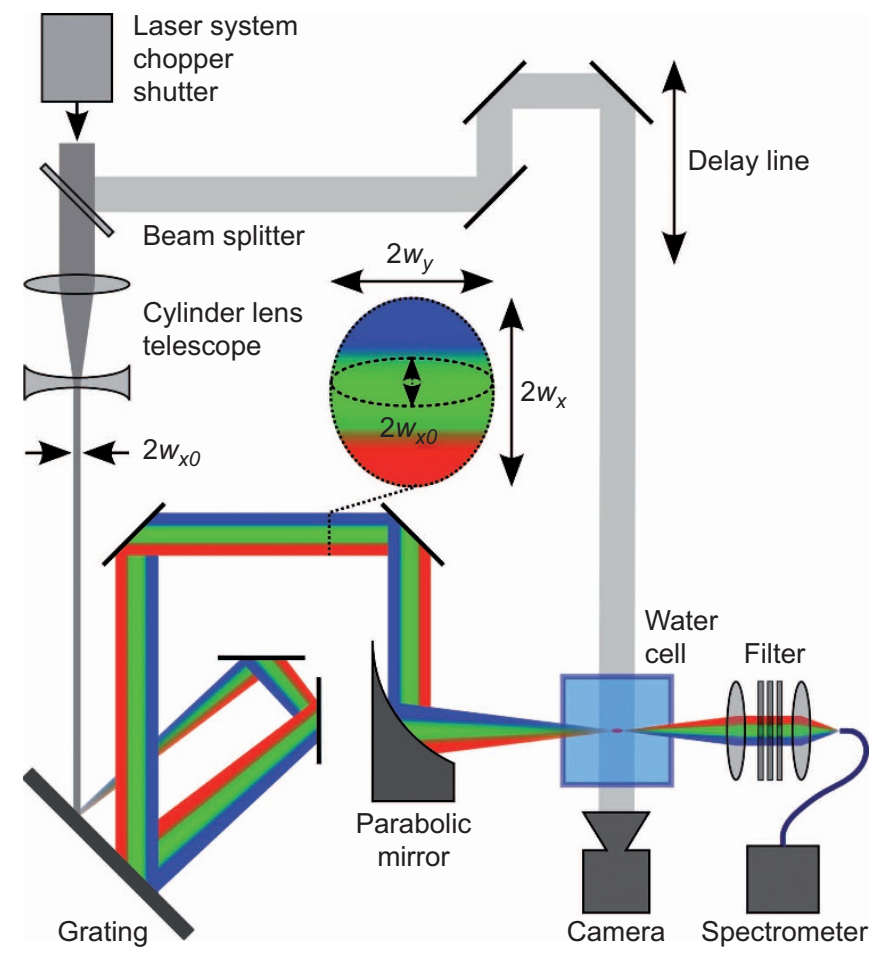

Figure 1 Sketch of the experimental pump-probe set-up to induce and observe the optical breakdown in a water cell by simultaneous spatial and temporal focusing. 
and collimated using a double-pass diffraction grating stretcher. The reflection grating (1500 lines $\mathrm{mm}^{-1}$ ) and incident angle were chosen to be equal to the compressor grating set-up of the chirped pulse amplification laser system to minimize third-order dispersion. Second-order dispersion induced by the SSTF grating configuration is compensated by pre-chirping the incident laser pulse by optimizing the chirped pulse amplification laser compressor gratings. ${ }^{35}$

The SSTF grating configuration expands the elliptical beam horizontally to a beam with a width of $2 w_{x}=5.4 \mathrm{~mm}$, consisting of spectrally separated beamlets (see inset in Figure 1). Because the vertical beam size is kept at $2 w_{y}=5.1 \mathrm{~mm}$, the spectrally separated beam has an approximately circular shape prior to focusing by means of a $90^{\circ}$ offaxis parabolic mirror (effective focal length $25.4 \mathrm{~mm}$, NT47-096; Edmund Optics, Barrington, NJ, USA). In accordance with the spectral separation, an autocorrelation of the partially blocked SSTF beam ${ }^{31}$ yielded a pulse duration of $\sim 300$ fs within the collimated beam prior to focusing. Moreover, the measurement revealed a minimal pulse duration of $\sim 55$ fs achievable at the focal spot of the SSTF configuration, similar to the initial pulse duration of the laser system $\left(\tau_{0}=50 \mathrm{fs}\right)$.

For purposes of investigating the optical breakdown induced by conventional focusing, the cylindrical telescope and the grating configuration are bypassed and the fs-pulses are focused directly. The resulting NA of the focusing optics in both regimes is $N A \approx 0.1$. The focal spot size is characterized using a high-resolution CMOS (complementary metal oxide semiconductor) camera sensor (pixel pitch $1.54 \mu \mathrm{m}$; Pentax Q, Ricoh Imaging Co., Ltd., Tokyo, Japan), which is moved along the propagation direction using a motorized translation stage (M-126 PD1; PI Physik Instrumente GmbH \& Co. KG, Karlsruhe, Germany) to detect the $x-y$ intensity distribution at the focal plane.

To investigate laser-induced optical breakdown simulating the treatment of the lens or the vitreous body of the eye, pulses are focused $15 \mathrm{~mm}$ deep into a cell (704.002-OG; Hellma GmbH \& Co. KG, Müllheim, Germany) filled with distilled water. To observe the plasma and disruption formation, the focal area is illuminated by probe pulses, which can be delayed up to 10 ns relative to the pump pulse. Shadowgraphic images are acquired using a $10 \times$ microscope objective (NT36-132; Edmund Optics Inc., Barrington, NJ, USA) and a CMOS camera (Thorlabs DCC1645M; Thorlabs Inc., Newton, NJ, USA). The image acquisition rate is synchronized with the chopper frequency $(10 \mathrm{~Hz})$.

For a quantitative breakdown analysis, artifacts are reduced by subtracting a reference image with the pump beam path blocked. After brightness normalization, the images are binarized to determine the full-width at half-maximum plasma length and diameter.

To analyze the plasma density, the temporal plasma evolution was observed by shifting the probe-pulse delay in steps of $\sim 170$ fs to detect the maximum absorbance. When observing the optical breakdown in water, free electrons are the main reason for absorbance within the first few hundred fs after ionization. ${ }^{21,36}$ Afterward, the absorbance decreases due to recombination and diffusion, whereas a significant amount of electrons $(20 \%-30 \%)$ is solvated and trapped by the water molecules due to their high polarity. This trapping causes longerlasting absorbance because the solvated states exhibit broad optical resonances in the near infrared and decay times $>500$ ps. Because the losses caused by the reflectivity of the plasma are weak $(\sim 0.01 \%)$ due to the low refractive index contrast of the plasma $\left(<10^{-3}\right)$ and the small probe pulse incidence angle, the absorbance of the free and solvated electrons is the main reason for the darkening in the shadowgraphic images. ${ }^{37}$ Thus, the average plasma density $n_{\mathrm{e}}$ directly after the pump pulse incidence can be estimated by $n_{\mathrm{e}} \approx \alpha / \sigma$, where $\alpha$ is the absorption coefficient of the plasma and $\sigma=1.44 \times 10^{-18} \mathrm{~cm}^{2}$ is the absorption cross-section for an electron collision time $\tau_{\mathrm{c}}=10 \mathrm{fs}$ according to the Drude model. ${ }^{38}$

The SC generated by the pulse within the water cell is collimated and characterized by two CCD (charge coupled device) spectrometers (USB 2000 \& USB 4000; Ocean Optics Inc., Dunedin, FL, USA), which allow single shot measurements of the visual (350-850 nm) and near infrared (750-1050 nm) spectra, respectively. For shorter or longer wavelengths, the transmission of the optical components, the limited spectrometer sensitivity and the low SC intensity impede a reliable single-shot detection. At each pulse energy and wavelength range, 100 single-shot measurements are acquired. The measurements are averaged and calibrated by the neutral density and color filter transmission and by the spectrometer sensitivity. After normalization, the measurements of the visual $(<800 \mathrm{~nm})$ and infrared $(>800 \mathrm{~nm})$ spectra are combined to characterize the entire spectral broadening.

\section{Numerical simulations}

In our numerical simulations, we solve the so-called standard model for fs filamentation, ${ }^{17,39}$ adapting the model to water as the nonlinear medium: ${ }^{16,40}$

$$
\begin{gathered}
\frac{\partial}{\partial z} \hat{E}=i \sqrt{k^{2}(\omega)-k_{x}^{2}-k_{y}^{2}} \hat{E}+i \frac{\mu_{0} \omega^{2}}{2 k(\omega)}\left(\hat{P}_{N L}+\frac{i}{\omega} \hat{J}_{e}\right)-\hat{L}_{I O N} \\
P_{N L}(t)=\frac{4 \varepsilon_{0} n_{0}^{2}}{3 \mu_{0} c} n_{e} E^{3}(t) ; \quad \frac{\partial}{\partial t} J_{e}+\frac{1}{\tau_{c}} J_{e}=\frac{e^{2}}{m_{e}} n_{e} E \\
L_{I O N}=\frac{U_{i} W(I) n_{n t}}{2 I} E ; \quad \frac{\partial}{\partial t} n_{e}=W(I) n_{n t}+\frac{\sigma I}{U_{i}} n_{e}-a n_{e}^{2}
\end{gathered}
$$

Here, the symbol ^ denotes the usual Fourier transform with respect to time $t$ and transverse spatial coordinates $x$ and $y$. The main approximation in the derivation of this model is the description of the laser pulse by a scalar forward-running field only, i.e., any backward-scattered fields are negligible. In the experimental study, linearly polarized and moderately focused $(\mathrm{NA} \approx 0.1)$ laser pulses are employed to generate plasma densities $n_{\mathrm{e}}<5 \times 10^{20} \mathrm{~cm}^{-3}$ well below the neutral density $n_{\mathrm{nt}}=3.3 \times 10^{22} \mathrm{~cm}^{-3}$, which justifies the above assumptions. Linear diffraction and dispersion are rigorously included in our fully space- and time-resolved $(3 \mathrm{D}+1)$ numerical code, and we use the tabulated values of the complex susceptibility $\chi(\omega)$ of water. Then, $k^{2}=\left(\omega^{2} / c^{2}\right)(1+\chi)$, and $n_{0}$ accounts for the refractive index at $800 \mathrm{~nm}$. Third-order nonlinear polarization is included via the Kerr coefficient $n_{2}=1.9 \times 10^{-16} \mathrm{~cm}^{2} \mathrm{~W}^{-1}$, while both nonlinear dispersion and higher order nonlinear susceptibilities are neglected. ${ }^{16}$ Note that our code rigorously accounts for third harmonic generation; however, due to the strong phase mismatch, the third harmonic spectral amplitude stays more than four orders of magnitude below the fundamental one and is not discussed in this paper. Ionization is included via the Keldysh theory, ${ }^{41}$ where we assume a gap potential of $U_{\mathrm{i}}=7 \mathrm{eV}$. In addition to the intensity-dependent Keldysh ionization rate $W(I)$, the plasma model is governed by avalanche ionization and the quadratic recombination with coefficient $a=2 \times 10^{-24} \mathrm{~cm}^{3} \mathrm{fs}^{-1}$.

To mimic the experimental pulse profiles while keeping the computational time within reasonable limits, the nonlinear propagation is started $2 \mathrm{~mm}$ before the linear focus. In the case of conventional focusing, a simple Gaussian pulsed beam with a radius of $w_{0}=200 \mu \mathrm{m}$ and a duration of $\tau_{0}=50 \mathrm{fs}$ is assumed and is focused by a perfect lens with a focal length $f=2 \mathrm{~mm}(\mathrm{NA} \approx 0.1)$. In the SSTF configuration, an elliptical pulsed beamlet with $w_{x 0}=40 \mu \mathrm{m}, w_{y}=200 \mu \mathrm{m}, \tau_{0}=50 \mathrm{fs}$ and a spatial 
chirp rate re $^{35} .9 \mathrm{~mm}$ fs in the $x$ direction is employed. Note that due to the spatial chirp rate, the resulting intensity profile at $z=-2 \mathrm{~mm}$ is perfectly symmetrical in the transverse $x-y$ plane, with a $1 / e^{2}$ intensity radius of $200 \mu \mathrm{m}$, as for conventional focusing, but the pulse duration is increased by a factor of 5 to $250 \mathrm{fs}$.

\section{RESULTS AND DISCUSSION}

\section{Experimental results}

For conventional focusing, the measurement of the intensity distribution at the focal plane in air yields a circular focal spot diameter of $\sim 7 \mu \mathrm{m}\left(1 / e^{2}\right)$, as shown in Figure 2a. Regarding SSTF, the size of the spectrally separated beam prior to focusing is equivalent $\left(2 w_{x, y}\right.$ $\sim 5 \mathrm{~mm}$ ), thus leading to a comparable NA and beam propagation toward the focal plane. Near the geometrical focus, the interference of the spectrally separated beamlets leads to the local shortening of the pulse duration. Furthermore, previous studies on SSTF have shown that a tilt of the pulse-front is induced near the focus, ${ }^{26,42}$ which leads to a sweep of the focal spot across the focal plane in $x$ direction. As the focal spot sweeps in a much faster time than the image acquisition system can resolve, the focal spot appears elliptical, as shown in Figure 2b.

For a detailed understanding of the breakdown dynamics, Figure $3 a-3 e$ presents time-resolved shadowgraphic images of the temporal plasma evolution in water for conventionally focused pulses of $3 \mu \mathrm{J}$. The transmission and the average plasma density of the central breakdown area (marked in Figure 3a-3e) are shown in Figure 3f. Right after the pump pulse incidence $(t=0)$, the absorption of the probe pulse increases, indicating the onset of plasma formation (Figure $3 \mathrm{a}-3 \mathrm{c}$ ). The maximum absorbance is reached within $\sim 0.5$ ps (Figure 3c), in good accordance with previous studies on plasma dynamics in water. ${ }^{36,43}$ Based on the simple assumption of a homogeneous plasma distribution within the plasma channel with a measured average diameter of $8 \pm 1 \mu \mathrm{m}$, we estimate an average plasma density of $(4 \pm 1) \times 10^{20} \mathrm{~cm}^{-3}$, according to the reduction in transmission at the location marked in Figure 3. Then, the plasma density decreases due to the recombination and diffusion of the free electrons, while longer-lasting absorbance is caused by solvated electrons.

Shadowgraphic images of the plasma induced by conventional focusing and SSTF are shown in Figure $4 \mathrm{a}$ and $4 \mathrm{~b}$. The probe-pulse delay was optimized for maximum average plasma contrast after pump pulse incidence. Figure $4 \mathrm{c}$ and $4 \mathrm{~d}$ illustrates the beginning of the disruptions due to vaporization at $t=10 \mathrm{~ns}$ after the pump pulse incidence.

Regarding conventional focusing, the threshold for visible plasma formation on the shadowgraphic images is $E_{\text {thres }} \sim 0.2 \mu \mathrm{J}$ or $I$ $\sim 6 \times 10^{12} \mathrm{~W} \mathrm{~cm}{ }^{-2}$, which is in good agreement with the breakdown thresholds in water determined by previous studies. ${ }^{44,45}$ Up to $2 \mu \mathrm{J}$, the plasma volume appears homogeneous and well confined to the focal plane, reaching a length of $\sim 350 \mu \mathrm{m}$ and a diameter of $\sim 6 \mu \mathrm{m}$
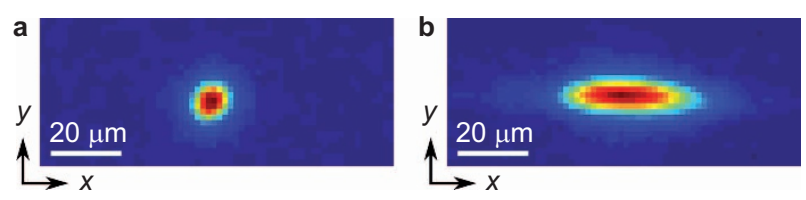

Figure 2 Intensity distribution at the focal plane $(z=0)$ for (a) conventional focusing and (b) SSTF configuration. SSTF, simultaneous spatial and temporal focusing.
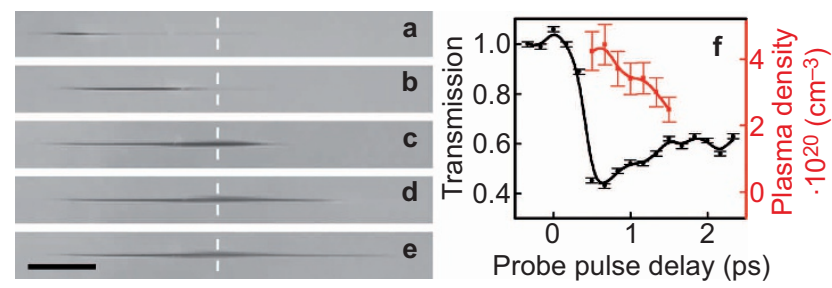

Figure 3 Temporal evolution of the plasma formation induced by conventionally focused pulses of $3 \mu \mathrm{J}$ : (a-e) present shadowgraphic images for increasing the probe-pulse delay ( $\Delta t=0.5 \mathrm{ps}$ ), beginning at $t=-0.5 \mathrm{ps}$ in a. Pump pulses incident from the left. Black scale bar indicates $100 \mu \mathrm{m}$. The white line marks the position where the probe-pulse transmission and plasma density are analyzed and plotted in (f).

(Figure 4a). Thus, disruptions recorded $10 \mathrm{~ns}$ after the pump pulse incidence also appear well localized (Figure 4c). Next to the central breakdown, brighter lines display shockwaves, which are emitted as a result of the fast thermal expansion of the breakdown volume. ${ }^{46}$ Using an intermediate pulse energy of $4 \mu \mathrm{J}$, the contrast of the shadowgraphic plasma images starts to become inhomogeneous for conventional focusing, indicating variations of the plasma density along the focal volume. Due to the inhomogeneous energy deposition, the formation of additional disruptions next to the optical axis is observed. For a high pulse energy of $8 \mu \mathrm{J}$, conventional focusing induces strong filamentation and the formation of several plasma side branches. Furthermore, the central plasma filament even exceeds the image area, with a length of $>1 \mathrm{~mm}$. Due to the formation of lateral filaments, the average vertical plasma diameter becomes $>13 \mu \mathrm{m}$. Additionally, the strongly inhomogeneous contrast within the central plasma filament indicates significant variations in the plasma density. As a consequence, the subsequent disruption breaks up into a multitude of smaller disruption volumes because the plasma density locally drops below the threshold of disruption formation.

For SSTF, the threshold for visible plasma formation is slightly increased to $E_{\text {thres }} \sim 0.4 \mu \mathrm{J}$ because of the slight increase of the focal pulse duration (55 fs $v s .50 \mathrm{fs}$ ) and the elliptically enlarged focal spot (Figure 2b). Because the breakdown area is observed in the vertical $y-z$ plane, the horizontal widening of the plasma volume is not imaged in our experiment. However, in contrast to conventional focusing, plasma formation is well localized to the focal plane and does not show the formation of extended filaments, additional side branches or inhomogeneities, even at high pulse energies up to $8 \mu \mathrm{J}$ (Figure $4 \mathrm{~b}$ ). The threshold of plasma formation is only exceeded within the focal volume, leading to a more homogeneous energy deposition and reducing the length and the vertical diameter of the plasma volume by a factor of 2. Thus, a plasma channel with a length of $<500 \mu \mathrm{m}$ and an average vertical diameter of $7 \mu \mathrm{m}$ is detected when focusing pulses with $8 \mu \mathrm{J}$ using SSTF. We would like to recall here that in the case of SSTF, the laser pulses are largely pre-chirped ( $\left.\tau_{\text {probe-pulse }}>10 \mathrm{ps}\right)$ to compensate for the dispersion induced by the SSTF set-up, which prevents the time-resolved detection of the maximum absorption and, thus, the estimation of the plasma density.

Figure $4 \mathrm{~d}$ presents shadowgraphic images of the disruptions within the water cell $10 \mathrm{~ns}$ after the incidence of the SSTF pump pulses. Similar to the homogeneous plasma formation, the subsequent disruptions are well confined to the focal plane. In contrast to conventional focusing, there is no splitting of the disruption volume. While the break-up of the disruptions leads to inhomogeneous shockwave emission in conventional focusing, the shockwaves generated by SSTF are more uniform. This uniformity is of special interest regarding 

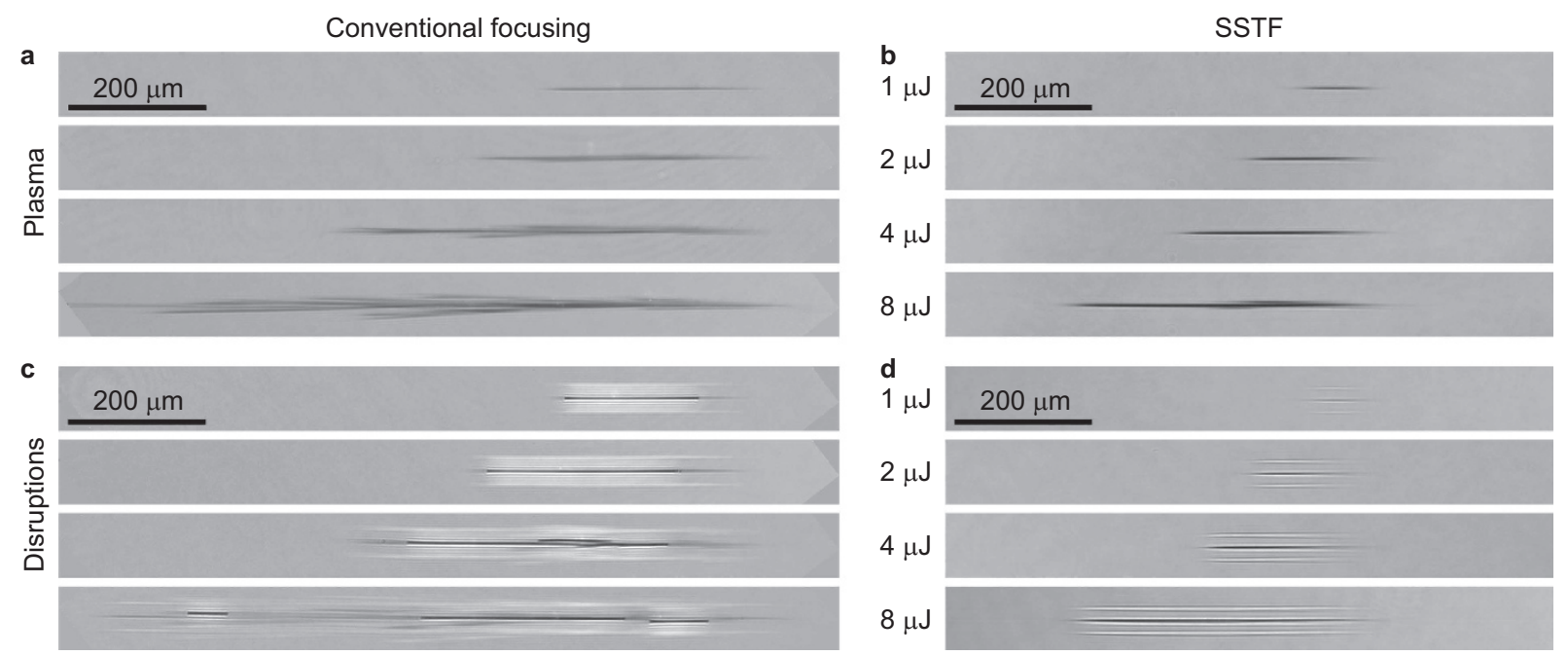

Figure 4 Shadowgraphic images of the plasma formation (a, b) and subsequent disruptions (c, d), illustrating the strongly confined optical breakdown and reduced filamentation effects in the SSTF configuration (right column, b, d) compared to conventional focusing (left column, a, c). Pump pulses incident from the left. SSTF, simultaneous spatial and temporal focusing.

fs-surgery because both the bubble formation by vaporization and the emitted shockwaves may damage adjacent cells. ${ }^{7}$

\section{Numerical analysis}

For a detailed understanding of plasma formation in SSTF and conventional focusing, Figure 5 presents snapshots of the simulated space-time $x / t$ intensity profiles $(y=0)$ for linear and nonlinear $\left(E_{\text {pulse }}=4 \mu \mathrm{J}\right)$ pulse propagation, illustrating both focusing conditions. For conventional focusing, the linear pulse propagation is symmetrical to the focal plane, showing the well-known converging and diverging behavior (Figure $5 \mathrm{a}-5 \mathrm{c}$ ). For higher pulse energies, nonlinear pulse propagation leads to a complex interplay between the Kerr self-focusing and defocusing effects of the induced plasma (Figure 5d-5f). Because the divergence of the pulse is hampered downstream from the focal plane, the intensity is kept trapped within a central region near the optical axis.

In contrast to conventional focusing, the spatiotemporal coupling mechanism responsible for pulse compression at the focus for the SSTF configuration is clearly visible in Figure $5 \mathrm{~g}-5 \mathrm{i}$ (linear regime). Outside the focal plane $(z= \pm 0.2 \mathrm{~mm})$, the spectral components are separated, leading to a pulse duration of $\sim 220 \mathrm{fs}$. At the focal plane (Figure 5h), the spectral overlap leads to a local shortening of the pulse to $\tau=50 \mathrm{fs}$. However, the spatiotemporal character of this pulse shortening manifests itself in the abovementioned characteristic pulse front tilt. ${ }^{28,42}$ At any position in the transverse $(x, y)$ plane, the pulse duration is short (50 fs), whereas the spatially integrated power profile of the pulse still has the initial duration of 250 fs for the single beamlets. In the nonlinear regime (Figure $5 \mathrm{j}-5 \mathrm{l}$ ), a strong distortion of the pulse profile is visible. However, the basic features of SSTF survive, leading to a broader intensity distribution before and behind the focus compared to conventional focusing. Although a similar maximum intensity of $I_{\max } \sim 24 \mathrm{TW} \mathrm{cm}^{-2}$ is reached at the focal plane $(z=0)$ for both focusing conditions, SSTF strongly suppresses the filamentation effect. Thus, $200 \mu \mathrm{m}$ downstream from the focal plane, the maximum intensity is already significantly decreased by a factor of $>10$ compared to conventional focusing.

To illustrate the resulting plasma formation caused by the simulated intensity distributions, Figure 6 presents iso-electron density surface
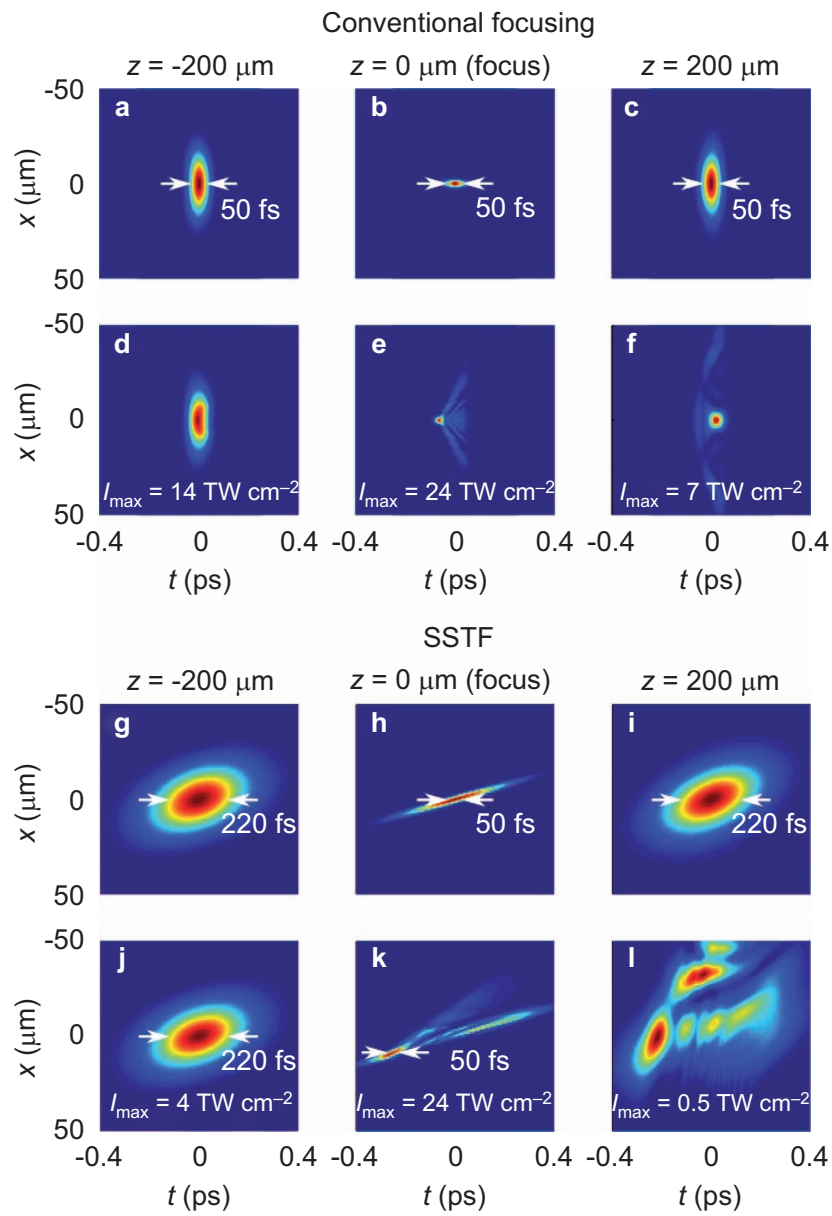

Figure $\mathbf{5}$ Snapshots of linear ( $\mathbf{a}-\mathbf{c}$ and $\mathbf{g}-\mathbf{i}$ ) and nonlinear pulse propagation $(\mathbf{d}-\mathbf{f}$ and $\mathbf{j}-\mathbf{I}, E_{\text {pulse }}=4 \mu \mathrm{J}$ ) for conventional focusing (upper section) and SSTF (lower section), showing $x / t$ intensity profiles $(y=0)$ at the indicated distances relative to the focus. Color scales are normalized to the individual maximum intensities of the subfigures. SSTF, simultaneous spatial and temporal focusing. 

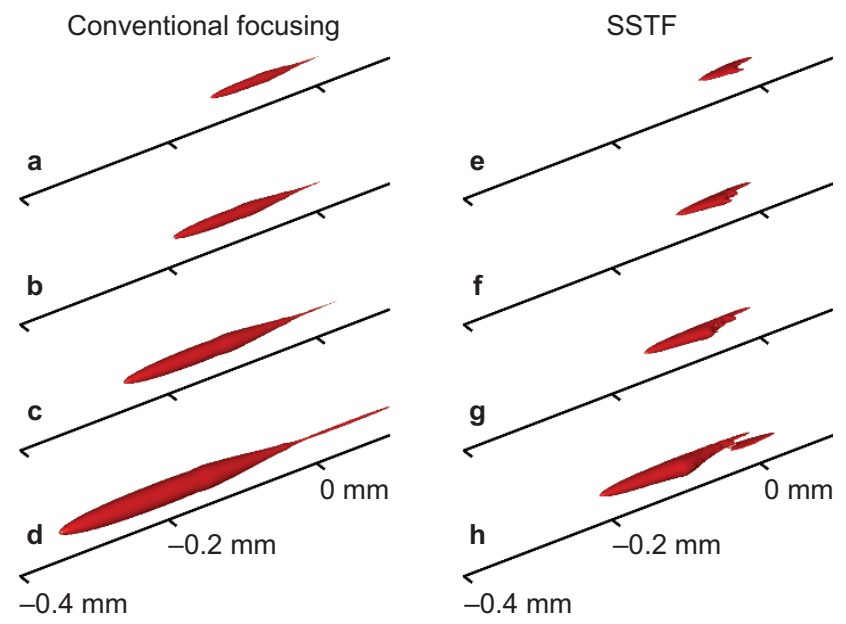

Figure 6 Simulation of plasma channels induced by conventional focusing (left column, (a-d)) and SSTF (right column, (e-h)) at $1 \mu \mathrm{J}, 2 \mu \mathrm{J}, 4 \mu \mathrm{J}$ and $8 \mu \mathrm{J}$, respectively. Iso-electron density surface plots are shown at $10^{19} \mathrm{~cm}^{-3}$. SSTF, simultaneous spatial and temporal focusing.

plots at $10^{19} \mathrm{~cm}^{-3}$ for different pulse energies and both focusing configurations. As in the experimental study on breakdown formation, the plasma channels induced by conventionally focusing low pulse energies $\leqslant 2 \mu \mathrm{J}$ are well localized. However, self-focusing can be observed even here, shifting the breakdown volume toward the focusing lens. For pulse energies $\geqslant 4 \mu \mathrm{J}$, the formation of a central filament strongly increases the length of the induced plasma (Figure $6 \mathrm{c}$ and $6 \mathrm{~d}$ ).

In SSTF, the formation of a central filament is impeded due to the sharply confined intensity distribution, therefore leading to a welllocalized plasma formation in propagation direction (Figure 6e-6h). For example, the plasma channel produced by the $8 \mu$ JSTF pulse (Figure $6 \mathrm{~h}$ ) has a similar length as the channel produced by the $2 \mu \mathrm{J}$ conventional pulse (Figure 6b). At the same time, inspection of the simulation data reveals that the energy deposited by the $8 \mu \mathrm{J}$ SSTF pulse is more than three times larger than that deposited by the $2 \mu \mathrm{J}$ conventional pulse. The maximum plasma densities achieved are in the range of $(2-4) \times 10^{20} \mathrm{~cm}^{-3}$, in excellent agreement with the estimates from our experiments. Interestingly, this value does not depend on the pulse energy, provided that the threshold energy for plasma formation is exceeded. The reason for this behavior is the well-known intensity clamping effect in femtosecond filaments: ${ }^{17,39}$ the maximum plasma densities and maximum intensities are solely determined by an inversion of the sign in the nonlinear induced refractive index change. As a consequence, all pulse intensities, whether conventionally focused or in the SSTF configuration, saturate at approximately $25 \mathrm{TW} \mathrm{cm}$.

Because we use ideal focusing conditions in our simulations, the resulting plasma channels are shorter than in the experiments (Figure 4). Moreover, due to the perfectly Gaussian beam shapes and the absence of aberrations, which certainly distort the pulse propagation in the experiment, no multifilamentation occurs in the numerical simulations. ${ }^{47}$ Consequently, the diameter of the plasma channel does not differ much for conventional focusing and SSTF. However, the experimentally observed overall reduction of the length of the plasma channel by a factor of 2 due to SSTF is nicely reproduced in our simulations.

\section{SC generation}

In addition to the plasma formation, the interaction of the intense laser pulse with the nonlinear medium leads to the generation of a broadband SC. The measurement and simulation results of SC induced by conventional focusing and SSTF are compared in Figure 7. For conventional focusing, a significant broadening of the pulse spectrum is already observed for the pulse energies $\leqslant 1 \mu \mathrm{J}$. With increasing pulse energy, the generated SC becomes broader and stretches toward shorter wavelengths (Figure 7a). Up to pulse energies of $4 \mu \mathrm{J}$, the maximum blue shift increases. For pulse energies $>4 \mu \mathrm{J}$, a cutoff at $\sim 400 \mathrm{~nm}$ is observed. Further increase of the pulse energy results only in an increased intensity of the broadened spectrum, as similarly reported by Liu et al., ${ }^{48}$ even for higher pulse energies up to $200 \mu \mathrm{J}$. Plasma defocusing clamps both the intensity and the wavelength shift to a maximum value because additional plasma filaments are formed due to the modulation instabilities of the laser pulse, as shown in Figure 4a.

Regarding SSTF, the spectral broadening of the incident laser pulse is strongly decreased (Figure 7b). For low pulse energies up to $2 \mu \mathrm{J}$, almost no frequency conversion is observed, and only a slight broadening is detected even for high pulse energies up to $8 \mu \mathrm{J}$.

The simulated spectra reproduce the strong decrease of SC in the SSTF configuration (Figure 7d). However, the spectra obtained for conventional focusing (Figure 7c) are much narrower than are their experimental counterparts. This discrepancy is readily explained by the differing nonlinear interaction lengths, as the experimental pump pulses propagate a longer distance of $15 \mathrm{~mm}$ within the water cell. Self-phase modulation as the main driver for SC generation is a cumulative effect, and a shorter channeling range should therefore reduce the SC generation.

In this context, we would like to comment on an interesting observation that once more illustrates certain peculiarities of SSTF pulses. When analyzing the measured or simulated spectra in Figure 7 with respect to plasma formation in Figures $4 \mathrm{a}, 4 \mathrm{~b}$ and 6 , one may wonder why SC is more pronounced for conventional focusing, even when compared to SSTF pulses with higher energies and comparable plasma length. For example, a $2 \mu \mathrm{J}$ pulse with conventional focusing produces a much broader spectrum than an $8 \mu \mathrm{J}$ SSTF pulse, though the length of their respective plasma channels is almost the same. The reasons for this initially surprising behavior are the differences in the actual (spatio-)temporal pulse shape. We noted above that the spectral broadening is mainly driven by self-phase modulation, which crucially depends on the pulse duration: short pulses modulate the phase much
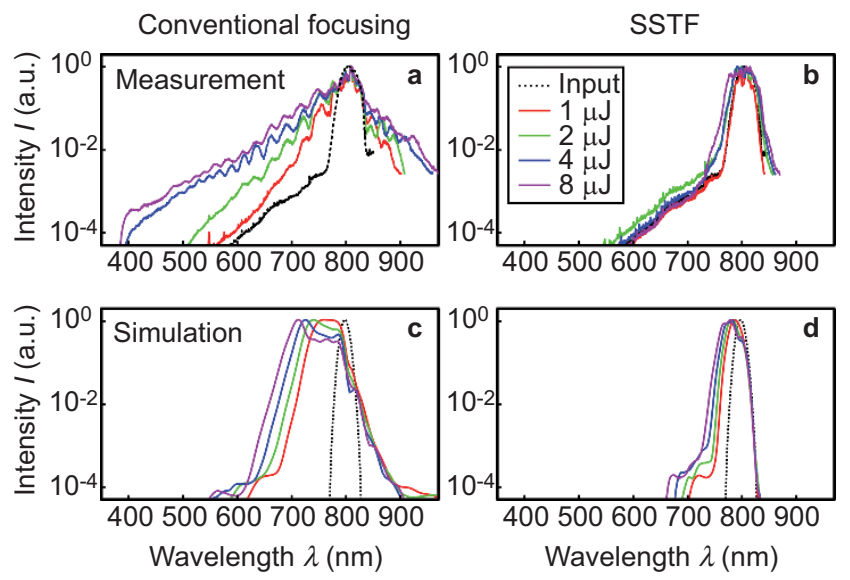

Figure 7 Experimental $(\mathbf{a}, \mathbf{b})$ and numerical $(\mathbf{c}, \mathbf{d})$ characterization of the supercontinuum generation in water, induced by conventional focusing (left column) and SSTF (right column). The black dotted lines indicate the input pulse spectra. SSTF, simultaneous spatial and temporal focusing. 
more strongly than longer pulses. For conventional focusing, the pulse envelope is always short, here 50 fs or even less. In the SSTF configuration, however, the pulse is short only in the vicinity of the focus (see also Figure 5), while slightly before or after the focus, the pulse duration is increased considerably and the self-phase modulation is strongly suppressed.

\section{CONCLUSIONS}

In conclusion, SSTF was investigated with the aim of improving the nonlinear processing of transparent materials using fs-laser pulses. Certain applications require optical breakdown formation by focused pulses of several $\mu \mathrm{J}$ and limited NA. Thus, nonlinear side effects such as self-focusing, filamentation, the break-up of disruptions and strong SC generation may reduce the precision and quality of the induced modifications. This drawback is of particular importance, e.g., in ophthalmic surgery: to fragment the cataractous lens tissue prior to extraction or to cut the vitreous body, pulse energies up to $10 \mu \mathrm{J}$ are applied, while the NA is limited by the pupil of the eye. ${ }^{9,10,14}$ Thus, filamentation reduces the quality of surgical cuts or may even harm the nearby capsular bag when using conventional focusing. Moreover, strong white-light generation poses a risk for retinal laser safety.

Here, simultaneous spatial and temporal focusing is applied for improved intensity confinement and a decrease in the nonlinear side effects. Because the ultrashort pulse is only formed at the focal plane in the SSTF configuration, the intensity outside the focal volume is strongly reduced, thus confining the laser-material interaction.

We have demonstrated this phenomenon by evaluating the optical breakdown and nonlinear side effects in a water cell, both experimentally and numerically comparing conventional focusing and SSTF. Regarding conventional focusing, pump-probe shadowgraphy revealed strong nonlinear side effects, such as the formation of additional plasma side branches and extended plasma filaments reaching a length of $>1 \mathrm{~mm}$. Thus, the inhomogeneous plasma density caused by the complex interplay of self-focusing and plasma defocusing led to a break-up of the disruptions and strongly fluctuating shockwave emission. Furthermore, self-phase modulation caused a broadband SC reaching $<400 \mathrm{~nm}$. For SSTF, the improved intensity confinement in propagation direction significantly reduced the nonlinear side effects. In excellent agreement with the numerical simulations, the length of the plasma channels was reduced by a factor of 2, and no filamentation was observed, even for high pulse energies up to $8 \mu \mathrm{J}$. Because no plasma side branches or axial inhomogeneities were induced, the subsequent disruptions were well confined to the focal volume. Moreover, white light generation through SC could be drastically reduced by SSTF. Only slight spectral broadening was observed experimentally or in the simulations, even for high pulse energies.

Consequently, the confined laser-material interaction and strongly reduced nonlinear side effects make SSTF a versatile technique for the high-precision processing of transparent materials, such as ophthalmic fs-surgery, where the restricted breakdown size and the impeded SC generation ensure precise and gentle treatment.

\section{CONTRIBUTIONS}

The presented work was carried out in collaboration among all authors. RK, RA and SN designed the experimental methods, and RK performed the experimental investigations and the data analysis. The numerical simulations were performed by JG and SS. RK and SS wrote the manuscript. The interpretation and discussion of the results were supported by JT and AT.

\section{ACKNOWLEDGMENTS}

This study was supported by the Thuringian Ministry of Education, Science and Culture (OptiMi 2020-Graduate Research School 'Green Photonics', B51410061), the German Research Foundation (Leibniz program) and the Carl Zeiss Foundation. The numerical simulations were performed at the Rechenzentrum Garching.

1 Cheng J, Liu CS, Shang S, Liu D, Perrie W et al. A review of ultrafast laser materials micromachining. Opt Laser Technol 2013; 46: 88-102.

2 Chichkov BN, Momma C, Nolte S, von Alvensleben F, Tünnermann A. Femtosecond, picosecond and nanosecond laser ablation of solids. Appl Phys 1996; 63: 109-115.

3 Gattass RR, Mazur E. Femtosecond laser micromachining in transparent materials. Nat Photon 2008; 2: 219-225.

4 Itoh K, Watanabe W, Nolte S, Schaffer CB. Ultrafast processes for bulk modification of transparent materials. MRS Bull 2006; 31: 620-625.

5 Szameit A, Nolte S. Discrete optics in femtosecond-laser-written photonic structures. J Phys B 2010; 43: 163001.

6 Kymionis GD, Kankariya VP, Plaka AD, Reinstein DZ. Femtosecond laser technology in corneal refractive surgery: a review. J Refract Surg 2012; 28: 912-920.

7 Vogel A, Noack J, Hüttman G, Paltauf G. Mechanisms of femtosecond laser nanosurgery of cells and tissues. Appl Phys B 2005; 81: 1015-1047.

8 Soong HK, Malta JB. Femtosecond lasers in ophthalmology. Am J Ophthalmol 2009; 147: 189-197.

9 Roberts TV, Lawless M, Chan CC, Jacobs M, Ng D et al. Femtosecond laser cataract surgery: technology and clinical practice. Clin Exp Ophthalmol 2013; 41: 180-186.

10 Conrad-Hengerer I, Hengerer FH, Schultz T, Dick HB. Effect of femtosecond laser fragmentation of the nucleus with different softening grid sizes on effective phaco time in cataract surgery. J Cataract Refract Surg 2012; 38: 1888-1894.

11 Schumacher S, Oberheide U, Fromm M, Ripken T, Ertmer W et al. Femtosecond laser induced flexibility change of human donor lenses. Vision Res 2009; 49: 1853-1859.

12 Reggiani Mello GH, Krueger RR. Femtosecond laser photodisruption of the crystalline lens for restoring accommodation. Int Ophthalmol Clin 2011; 51: 87-95.

13 Ackermann R, Kunert KS, Kammel R, Bischoff S, Bühren SC et al. Femtosecond laser treatment of the crystalline lens: a 1-year study of possible cataractogenesis in minipigs. Graefes Arch Clin Exp Ophthalmol 2011; 249: 1567-1573.

14 Merker M, Ackermann R, Kammel R, Kunert KS, Nolte S. An in vitro study on focusing fs-laser pulses into ocular media for ophthalmic surgery. Lasers Surg Med 2013; 45: 589-596.

15 Heisterkamp A, Ripken T, Mamom T, Drommer W, Welling $\mathrm{H}$ et al. Nonlinear side effects of fs pulses inside corneal tissue during photodisruption. Appl Phys B 2002; 74: 419-425.

16 Skupin S, Nuter R, Bergé L. Optical femtosecond filaments in condensed media. Phys $\operatorname{Rev} A$ 2006; 74: 043813.

17 Couairon A, Mysyrowicz A. Femtosecond filamentation in transparent media. Phys Rep 2007; 441: 47-189.

18 Brodeur A, Chin SL. Ultrafast white-light continuum generation and self-focusing in transparent condensed media. J Opt Soc Am B 1999; 16: 637-650.

19 Ackermann R, Kammel R, Merker M, Kamm A, Tünnermann A et al. Optical sideeffects of fs-laser treatment in refractive surgery investigated by means of a model eye. Biomed Opt Express 2013; 4: 220-229.

20 Liu W, Kosareva O, Golubtsov IS, Iwasaki A, Becker A et al. Femtosecond laser pulse filamentation versus optical breakdown in $\mathrm{H}_{2} \mathrm{O}$. Appl Phys B 2003; 76: 215-229.

21 Minardi S, Gopal A, Tatarakis M, Couairon A, Tamošauskas G et al. Time-resolved refractive index and absorption mapping of light-plasma filaments in water. Opt Lett 2008; 33: 86-88.

22 Couairon A, Mysyrowicz A. Self-focusing and filamentation of femtosecond pulses in air and condensed matter: simulations and experiments. In: Boyd RW, Lukishova SG, Shen YR, editors. Self-focusing: past and present: fundamentals and prospects. Berlin: Springer; 2009. pp297-322.

23 Loesel FH, Niemz MH, Bille JF, Juhasz T. Laser-induced optical breakdown on hard and soft tissues and its dependence on the pulse duration: experiment and model. IEEE J Quantum Elect 1996; 32: 1717-1722.

24 Zhu G, van Howe J, Durst M, Zipfel W, Xu C. Simultaneous spatial and temporal focusing of femtosecond pulses. Opt Express 2005; 13: 2153-2159.

25 Oron D, Tal E, Silberberg Y. Scanningless depth-resolved microscopy. Opt Express 2005; 13: 1468-1476.

26 Durst ME, Zhu G, Xu C. Simultaneous spatial and temporal focusing in nonlinear microscopy. Opt Commun 2008; 281: 1796-1805.

27 Dana H, Kruger N, Ellman A, Shoham S. Line temporal focusing characteristics in transparent and scattering media. Opt Express 2013; 21: 5677-5687.

28 Vitek DN, Block E, Bellouard Y, Adams DE, Backus S et al. Spatio-temporally focused femtosecond laser pulses for nonreciprocal writing in optically transparent materials. Opt Express 2010; 18: 24673-24678.

29 Vitek DN, Adams DE, Johnson A, Tsai PS, Backus S et al. Temporally focused femtosecond laser pulses for low numerical aperture micromachining through optically transparent materials. Opt Express 2010; 18: 18086-18094.

30 Block E, Greco M, Vitek D, Masihzadeh O, Ammar DA et al. Simultaneous spatial and temporal focusing for tissue ablation. Biomed Opt Express 2013; 4: 831-841. 
31 Kammel R, Ackermann R, Tünnermann A, Nolte S. Pump-probe investigation of fsLIOB in water by simultaneous spatial and temporal focusing. Proc SPIE 2013; 8611: 86110A.

$32 \mathrm{He} \mathrm{F}, \mathrm{Xu} \mathrm{H}$, Cheng Y, Ni JL, Xiong $\mathrm{H}$ et al. Fabrication of microfluidic channels with a circular cross section using spatiotemporally focused femtosecond laser pulses. Opt Lett 2010; 35: 1106-1108.

33 Straub A, Durst ME, Xu C. High speed multiphoton axial scanning through an optical fiber in a remotely scanned temporal focusing setup. Biomed Opt Express 2011; 2: 80-88.

34 Durst ME, Zhu G, Xu C. Simultaneous spatial and temporal focusing for axial scanning. Opt Express 2006; 14: 12243-12254.

35 Durfee CG, Greco M, Block E, Vitek D, Squier JA. Intuitive analysis of space-time focusing with double-ABCD calculation. Opt Express 2012; 20: 14244-14259.

36 Sander MU, Gudiksen MS, Luther K, Troe J. Liquid water ionization: mechanistic implications of the H/D isotope effect in the geminate recombination of hydrated electrons. Chem Phys 2000; 258: 257-265.

37 Sun Q, Jiang H, Liu Y, Wu Z, Yang H et al. Measurement of the collision time of dense electronic plasma induced by a femtosecond laser in fused silica. Opt Lett 2005; 30: 320-322.

38 Stuart BC, Feit MD, Herman S, Rubenchik AM, Shore BW et al. Nanosecond-tofemtosecond laser-induced breakdown in dielectrics. Phys Rev B 1996; 53: 17491761.

39 Bergé L, Skupin S, Nuter R, Kasparian J, Wolf JP. Ultrashort filaments of light in weakly ionized, optically transparent media. Rep Prog Phys 2007; 70: 1633-1713.

40 Kolesik M, Wright EM, Moloney JV. Dynamic nonlinear X waves for femtosecond pulse propagation in water. Phys Rev Lett 2004; 92: 253901.

41 Keldysh LV. Ionization in the field of a strong electromagnetic wave. Sov Phys JETP 1965; 20: 1307-1314.
42 Coughlan MA, Plewicki M, Levis RJ. Parametric spatio-temporal control of focusing laser pulses. Opt Express 2009; 17: 15808-15820.

43 Migus A, Gauduel Y, Martin JL, Antonetti A. Excess electrons in liquid water: first evidence of a prehydrated state with femtosecond lifetime. Phys Rev Lett 1987; 58 : 1559-1562.

44 Feng Q, Moloney JV, Newell AC, Wright EM, Cook K et al. Theory and simulation on the threshold of water breakdown induced by focused ultrashort laser pulses. IEEE J Quantum Elect 1997; 33: 127-137.

45 Schaffer CB, Nishimura N, Glezer EN, Kim AM, Mazur E. Dynamics of femtosecond laser-induced breakdown in water from femtoseconds to microseconds. Opt Express 2002; 10: 196-203.

46 Vogel A, Linz N, Freidank S, Paltauf G. Femtosecond-laser-induced nanocavitation in water: implications for optical breakdown threshold and cell surgery. Phys Rev Lett 2008; 100: 038102.

47 Bergé L, Gouédard C, Schjødt-Eriksen J, Ward H. Filamentation patterns in Kerr media vs. beam shape robustness, nonlinear saturation and polarization states. Physica $D$ 2003; 176: 181-211.

48 Liu W, Petit S, Becker A, Akozbek N, Bowden CM et al. Intensity clamping of a femtosecond laser pulse in condensed matter. Opt Commun 2002; 202: 189-197.

(c) (i) (-) $\odot$ This work is licensed under a Creative Commons Attribution-

NonCommercial-NoDerivs 3.0 Unported License. The images or other third party material in this article are included in the article's Creative Commons license, unless indicated otherwise in the credit line; if the material is not included under the Creative Commons license, users will need to obtain permission from the license holder to reproduce the material. To view a copy of this license, visit http://creativecommons.org/licenses/by-nc-nd/3.0/ 